\title{
Probiotics: Should We Consider it in Therapeutics?
}

\author{
MD. ISMAIL PATWARY, ${ }^{1}$ MD. KHALILUR RAHMAN²
}

\begin{abstract}
:
Probiotics are receiving increasing attention in the medical field. This is partly due to the recognition that microbial resistance to antibiotics presents a serious world-wide problem. In addition, fast growing insight into host-microbe interactions opened new routes in the development of rational alternatives in therapy and prevention of diseases as result of the complex procaryotic-eucaryotic evolutionary symphony. Consequently, long existing aspects of probiotics came into focus, and already provide exciting prospects.

Keyword: Probiotics, Microflora, Health benefits
\end{abstract}

\section{Introduction:}

The widely used definition, developed by the World Health Organization and the Food and Agriculture Organization of the United Nations, is that probiotics are "live microorganisms, which, when administered in adequate amounts, confer a health benefit on to the host." Lactic acid bacteria (LAB) and bifidobacteria are the most common types of microbes used as probiotics; but certain yeasts and bacilli may also be helpful. Probiotics are commonly consumed as part of fermented foods with specially added active live cultures; such as in yogurt, soy yogurt, or as dietary supplements. The human host and its microflora constitute a complex ecosystem whose equilibrium serves as a remarkable example of reciprocal adaptation. Some bacterial species that may colonize the gut are potentially harmful as a result of toxin production, mucosal invasion, or activation of carcinogens and inflammatory responses. ${ }^{1}$ Conversely, the indigenous, healthy intestinal flora provides resistance to colonization by these pathogenic microorganisms through several mechanisms.

\section{Microflora in the human intestine}

Establishment of the intestinal microflora begins at birth and progresses throughout life. The gastrointestinal (GI) tract is the most densely colonized region of the human body, with approximately $10^{12}$ bacteria per gram of contents in the large intestine. ${ }^{2}$ This complex ecosystem of intestinal microflora is estimated to contain approximately 500 different bacterial species of anaerobes as well as facultative and aerobic organisms, constituting a highly active society of organisms that can have both beneficial and detrimental influences on the host. ${ }^{1}$

The pathobiotics (harmful to the host) can modify the function of the intestinal epithelium to enhance their penetration across this barrier, serving as a link between inflammatory diseases of the gut and systemic inflammatory disorders. ${ }^{1,3}$ But the host friendly microflora play crucial role to fight against the offending organisms via different ways:

- Strengthening the epithelial barrier, thereby preventing translocation of pathogenic bacteria.

- Competing with pathogenic bacteria for attachment to epithelial cells.

- Producing antimicrobial compounds.

- Creating a local environment that is unfavorable for the growth of many pathogens.

- Enhancing the mucosal immune response to pathogens.

How the micro population of the intestine is affected?

There is a progressive increase in immune-mediated, gutrelated health problems such as allergies and autoimmune and inflammatory diseases in modern society. ${ }^{2}$ Many factors are theorized to contribute to a disruption of healthy bacterial balance and a decline in host defense mechanisms in the gut. These include medications such as gastric acid inhibitors or neutralizers, high fat/low fiber diets, and contaminated food and water. But perhaps the most prevalent cause of indigenous microflora disruption is the widespread and chronic use of antibiotics. ${ }^{4,5}$ Because antibiotics do not distinguish between pathogenic and healthful bacteria, they can severely disrupt intestinal and urogenital microbial balance. Suppression of the indigenous microflora may lead to reduced colonization resistance and overgrowth of preexisting, naturally resistant microorganisms, such as Clostridium difficile, which may cause diarrhea and/or colitis. ${ }^{4}$ For example, a recent study found that after a course of antibiotics, patients were 3 times as likely to report

1. Prof. \& Head, Department of Medicine, Sylhet MAG Osmani Medical College Hospital.

2. Student of MD (Internal Medicine), Final Part,Sylhet MAG Osmani Medical College Hospital.

Correspondence: Dr. Md. Ismail Patwary, Prof. \& Head, Department of Medicine, Sylhet MAG Osmani Medical College Hospital. 
functional bowel complaints 4 months after treatment compared with patients who did not receive antibiotics. ${ }^{6}$ Additionally, by altering the microflora of the vagina, antibiotics can increase a woman's propensity for urinary tract infections (UTIs) and vaginal candidiasis. ${ }^{7}$ New colonization by resistant pathogens may also occur, thus promoting the emergence and spread of antibiotic resistant strains. ${ }^{5}$ The injudicious overuse and misuse of antibiotics has led to a global emergence of antibiotic resistance. To illustrate, $70 \%$ of bacteria that cause hospital-acquired infections are resistant to at least one commonly used antibiotic, and some organisms are resistant to all approved antibiotics and must be treated with experimental drugs with potential toxicity. ${ }^{8}$

Community-acquired antibiotic resistance is also a growing problem, as evidenced by the fact that in some communities up to $40 \%$ of Streptococcus pneumoniae strains are now resistant to penicillin. ${ }^{9}$ Additionally, there is an increasing prevalence of resistance to several antibiotics used to treat UTIs. ${ }^{10}$

A well-balanced intestinal microflora reduces the risk of infections in the gut and urogenital tract, and thus is an important factor in reducing the need for antibiotics. Furthermore, a healthy microflora can prevent establishment of resistant strains. In this context, it is easy to see that if the microflora balance and colonization resistance of the host could be maintained at a healthy, normal level, the risk of emergence and spread of resistant strains could be mitigated. This may be accomplished through the therapeutic application of beneficial microorganisms, or probiotics.

\section{Probiotics- the promoters in restoring healthy microbes}

Probiotics are viable microorganisms that alter the microflora of the host and exert beneficial health effects. The concept of using beneficial flora for disease treatment and prevention as well as health restoration and maintenance is not new;fermented dairy products such as yogurt and milk have been used for hundreds of years to promote health. ${ }^{11}$ Today, the numerous health effects attributed to the use of probiotics are well documented and supported by modern science. ${ }^{1,3,11-29}$ These benefits include:

- Protection against infection by pathogenic bacteria

- Lower frequency and duration of diarrhea associated with antibiotic use.

- Improvement of lactose intolerance

- Stimulation of immune function

- Decreased fecal concentrations of enzymes, mutagens, and secondary bile salts that may be involved in colon carcinogenesis. Other benefits of probiotics currently under study include reduction of allergic symptoms and atopic dermatitis, relief from constipation, irritable bowel syndrome (IBS), and inflammatory bowel disease, improvement in mineral absorption, and lowering of cholesterol. ${ }^{11,30-32}$

\section{Probiotics selection criteria}

The criteria for a microorganism to be defined as a probiotic include that the strain be of human origin, be safe for human use, be stable in acid and bile, and adhere to the intestinal mucosa. ${ }^{21,33,34}$ The genera most frequently used as probiotics are Lactobacillus and Bifidobacterium. While many lactobacilli strains are available for human use, relatively few have substantial scientific evidence that supports their effectiveness. ${ }^{21,34,35}$ Furthermore, many questions have been raised about reliability, viable content, and the general quality of many probiotic products. The properties that are important in selecting a strain of lactobacilli include the ability to adhere to the intestinal mucosa, resist acid and bile, and produce antimicrobial substances.

\section{Intestinal Adherence}

The ability to adhere to the intestinal mucosa is a critical selection criteria because adhesion is considered to be a prerequisite for colonization. ${ }^{17}$ Adhesion is also considered important for stimulation of the immune system. The stomach and upper intestinal tract represent a hostile environment that can easily discourage growth or survival of microorganisms. Although many lactobacilli survive selective pressures of this environment, flow rates of digesta through the small intestine would wash out any organism unable either to multiply rapidly enough to avoid dilution or to maintain their residence by physical attachment to the intestinal epithelium. ${ }^{36}$

\section{Acid and Bile Tolerance}

To survive passage through the stomach and upper small intestine, probiotic strains must be able to tolerate the acidic and protease-rich environment of the stomach, and survive in the presence of bile acids. ${ }^{33}$ Bile tolerance is considered to be an important characteristic of $L$. acidophilus that enables it to survive, grow, and exert its action in the lower small intestine and colon. ${ }^{31,33,37}$

\section{Antimicrobial Activity}

The ability to competitively exclude or inhibit pathogenic intestinal bacteria through antimicrobial action is a major benefit attributed to L. acidophilus, and is an important consideration when selecting strains. ${ }^{38}$ These substances include growth-inhibiting metabolites such as organic acids and hydrogen peroxide, as well as bacteriocins, adhesion inhibitors, and a range of small antimicrobial substances. ${ }^{33}$ 


\section{Clinical Evidence of health benefits}

Numerous studies have confirmed the many wide-ranging beneficial effects of probiotics, such as improving intestinal tract health, enhancing the immune system, and reducing symptoms of lactose intolerance. In addition, evidence is emerging that potential benefits of probiotics may include treatment and prevention of allergies and inflammatory diseases, cancer prevention, and a reduction in respiratory disease. Several of these clinical benefits are discussed below.

\section{Anti-Carcinogenicity}

There is considerable interest in the role of the intestinal microflora in colon carcinogenesis ${ }^{12,16,19}$ and other forms of cancer. $^{25,39}$ Bacterial metabolism of various dietary constituents results in the production of many compounds, some of which may be carcinogenic. Altering the composition of the intestinal flora with probiotics may have a major effect on the production rate of these compounds. Indeed, epidemiological studies indicate a correlation between regular consumption of fermented dairy products and low incidence of colon cancer. ${ }^{12,19,25,39}$ Several studies have measured the activities of microbial enzymes that play a role in the conversion of procarcinogens to carcinogens, including glucuronidase, nitroreductase, and azoreductase. ${ }^{19,25,26,39}$

\section{Small Bowel Bacterial Overgrowth}

Patients with chronic kidney disease often suffer from small bowel bacterial overgrowth (SBBO), which is accompanied by production of toxic amines such as dimethylamine (DMA). ${ }^{17,28}$ The researchers found that Probiotics changed small bowel pathobiology by modifying metabolic actions of SBBO, reducing generation of toxins and carcinogens with no adverse side effects.

\section{Immune Function}

Approximately $70 \%$ of the immune system is localized in the GI tract and the intestinal mucosa provides a protective host defense against the constant presence of antigens from food and microorganisms. ${ }^{3,40}$ The intestinal microflora provides important support for this defense barrier, as shown by increased antigen transport across the gut mucosa in its absence. ${ }^{40}$ In addition to strengthening the intestine's permeability barrier, probiotics have been shown to enhance immune responses, particularly immunoglobulin $\mathrm{A}$, and thereby promote the intestine's immunologicbarrier. Probiotics have also been shown to stimulate non-specific host resistance to microbial pathogens and to control the balance of proinflammatory and antiinflammatory cytokines. ${ }^{40}$ Therefore, in addition to strengthening the intestinal barrier, probiotics may down-regulate hypersensitivity reactions and hold promise in the prevention and treatment of conditions associated with gut barrier dysfunction and sustained inflammatory responses, such as inflammatory bowel disease.

\section{Assimilation of Cholesterol}

It has been reported that $L$. acidophilus may play a role in reduced serum cholesterol levels in humans and animals.31,32 Certain strains of L. acidophilus, have the ability in vitro to assimilate cholesterol from a laboratory growth medium, although with wide variation. ${ }^{32,35}$ Such assimilation of cholesterol in the small intestine may be important in reducing the absorption of dietary cholesterol from the digestive system into the blood. ${ }^{31,32}$

\section{The Health benefits of Bifidobacteria}

Bifidobacteria is another probiotic naturally occurring in humans, with Bifidobacterium infantis being the dominant microorganism to colonize the intestines of breast-fed newborns. Research studies have documented several beneficial effects of bifidobacteria when given to infants, such as its effectiveness against a specific strain of enteropathogenic $E$. coli, in prevention of enteric infections, and in decreasing the growth of C. albicans. ${ }^{14,18,41}$ Recent research into the immune-enhancing effectiveness of bifidobacteria in the elderly also shows great promise. The researchers suggest that supplementation of bifidobacteriain the elderly may be a natural, non-invasive way to resist the decline in cellular immunity associated with the aging process. Acids produced by bifidobacteria include short-chain fatty acids (SCFAs) such as acetic, propionic, and butyric acids, as well as lactic and formic acids. ${ }^{41,42}$ These acids have an antimicrobial effect by lowering the $\mathrm{pH}$ of the colon, in addition to other inhibitory influences on bacterial cell growth. The most plentiful SCFA produced by bifidobacteria is acetic acid, which exerts a wide range of antimicrobial activity against yeasts and molds as well as bacteria.

\section{Conclusion:}

Old age, antibiotics and other medications, and immunocompromised states can all contribute to a disruption of the indigenous microflora population, putting patients at increased risk for infection and disease. The use of probiotics offers an intervention with essentially no risk that may provide significant health benefits by stabilizing the intestinal microflora. When choosing a probiotic, it is important to select strains that fulfill the criteria that are required for establishment in the intestinal tract and have demonstrated clinical effectiveness.

\section{Conflict of Interest : None}




\section{References:}

1. Isolauri E. Probiotics in human disease. Am J Clin Nutr 2001;73(supp1):114S-16S.

2. Klaenhammer TR. Probiotic bacteria: today and tomorrow. J Nutr 2000;130(2S Suppl):415S-16S

3. Lu L, Walker WA. Pathologic and physiologic interactions of bacteria with the gastrointestinal epithelium. Am J Clin Nutr 2001;73(suppl):1124S-30S.

4. Edlund C, Nord CE. Effect on human normal microflora of oral antibiotics for treatment of urinary tract infections. $\mathrm{J}$ Antimicrob Chemother 2000;46(Suppl 1):41-48.

5. Sullivan A, Edlund C, Nord CE. Effect of antimicrobial agents on the ecological balance of human microflora. Lancet Infect Dis $2001 ; 1(2): 101-14$.

6. Maxwell PR, Rink E, Kumar D, et al. Antibiotics increase functional abdominal symptoms. Am J Gastroenterol 2002;97(1):104-08.

7. Kurowski K, Ghosh R, Singh SK, et al. Clarithromycininduced alterations in vaginal flora. Am J Ther 2000;7(5):29195.

8. U.S. Food and Drug Administration: Antibiotic resistance. (n.d.). Retrieved March 27, 2002, from http://www.fda.gov/ oc/opacom/hottopics/anti_resist.html

9. Cassell GH, Mekalanos J. Antibiotic resistance. JAMA 2001;285:601-05.

10. Gupta K, Scholes D, Stamm WE. Increasing prevalence of antimicrobial resistance among uropathogens causing acute uncomplicated cystitis in women. JAMA 1999;281(8):73638 .

11. Schrezenmeir J, de Vrese M. Probiotics, prebiotics, and synbiotics - approaching a definition. Am J Clin Nutr 2001;73 (suppl):361S-64S.

12. Rao CV, Sanders ME, Indranie C, et al. Prevention of colonic preneoplastic lesions by the probiotic Lactobacillus acidophilus $\mathrm{NCFM}^{\mathrm{TM}}$ in $\mathrm{F} 344$ rats. Int $\mathrm{J}$ Oncol 1999; 14(5):939-44.

13. Dunn SR, Simenhoff ML, Ahmed KE, et al. Effect of oral administration of freeze-dried Lactobacillus acidophilus on small bowel bacterial overgrowth in patients with end stage kidney disease: reducing uremic toxins and improving nutrition. Int Dairy J 1998;8:545-53.

14. Wagner RD, Pierson C, Warner T, et al. Biotherapeutic effects of probiotic bacteria on candidiasis in immunodeficient mice. Infect Immun 1997;65(10):4165-72.

15. Reid G. In vitro testing of Lactobacillus acidophilus NCFM as a possible probiotic for the urogenital tract. Int Dairy $\mathrm{J}$ $2000 ; 10: 415-19$
16. Wollowski I, Rechkemmer G, Pool-Zobel BL. Protective role of probiotics and prebiotics in colon cancer. Am J Clin Nutr 2001;73(suppl):451S-55S.

17. Sanders ME, Klaenhammer TR. The scientific basis of Lactobacillus acidophilus NCFM functionality as a probiotic. J Dairy Sci 84:319-31.

18. Hoyos AB. Reduced incidence of necrotizing enterocolitis associated with enteral administration of Lactobacillus acidophilus and Bifidobacterium infantis to neonates in an intensive care unit. Int J Infect Dis 1999;3(4):197-202.

19. Goldin BR, Swenson L, Dwyer J, et al. Effect of diet and Lactobacillus acidophilus supplements on human fecal bacterial enzymes. J Natl Cancer Instit 1980;64:255-61.

20. Gilliland SE, Speck ML. Antagonistic action of Lactobacillus acidophilus toward intestinal and food borne pathogens in associative cultures. J Food Protection 1977;40(12):82023.

21. Schauss AG. Lactobacillus acidophilus: method of action, clinical application, and toxicity data. JAdv Med 1990;3(3): 163-78.

22. Lin MY, Savaiano D, Harlander S. Influence of nonfermented dairy products containing bacterial starter cultures on lactose maldigestion in humans. J Dairy Sci 1991;74:87-95.

23. Barefoot SF, Klaenhammer TR. Detection and activity of lactacin B, a bacteriocin produced by Lactobacillus acidophilus. Appl Environ Microbiol 1983;45:1808-15.

24. Barefoot SF, Chen YR, Hughes TA, et al. Identification and purification of a protein that induces production of the Lactobacillus acidophilus bacteriocin lactacin B. Appl Environ Microbiol 1994;60:3522-28.

25. Goldin BR, Gorbach SL. The effect of milk and Lactobacillus feeding on human intestinal bacterial enzyme activity. Amer J Clin Nutr 1984;39:756-61.

26. Goldin BR, Gorbach SL. Alterations of the intestinal microflora by diet, oral antibiotics, and Lactobacillus: decreased production of free amines from aromatic nitro compounds, azo dyes, and glucuronides. J Natl Cancer Instit 1984;73:689-95.

27. Goldin BR, Gorbach SL. Effect of Lactobacillus acidophilus dietary supplements on 1,2-dimethylhydrazine dihydrochloride- induced intestinal cancer in rats. J Natl Cancer Instit 1980;64:263-65.

28. Simenhoff ML, Dunn SR, Zollner GP, et al. Biomodulation of the toxic and nutritional effects of small bowel bacterial overgrowth in end-stage kidney disease using freeze-dried Lactobacillus acidophilus. Miner Electrolyte Metab 1996;22:92-96.

29. Kim GS, Gilliland SE. Lactobacillus acidophilus as a dietary adjunct for milk to aid lactose digestion in humans. J Dairy Sci 1983;66:959-66. 
30. Marteau PR, de Vrese M, Cellier CJ, et al. Protection from gastrointestinal diseases with the use of probiotics. Am J Clin Nutr 2001;73(suppl):430S-36S.

31. Walker DK, Gilliland SE. Relationships among bile tolerance, bile salt deconjugation, and assimilation of cholesterol by Lactobacillus acidophilus. J Dairy Sci 1993;76:956-61.

32. Gilliland SE, Nelson CR, Maxwell C. Assimilation of cholesterol by Lactobacillus acidophilus. Appl Environ Microbiol 1985;49:377-81.

33. Tuomola E, Crittenden R, Playne M, et al. Quality assurance criteria for probiotic bacteria. Am J Clin Nutr 2001;73 (suppl):393S-98S.

34. Reid G. The scientific basis for probiotic strains of Lactobacillus. Appl Environ Microbiol 1999;65(9):376366.

35. Gilliland SE, Walker DK. Factors to consider when selecting a culture of Lactobacillus acidophilus as a dietary adjunct to produce a hypocholesterolemic effect in humans. J Dairy Sci 1990;73:905-11.
36. Kleeman EG, Klaenhammer TR. Adherence of Lactobacillus species to human fetal intestinal cells. J Dairy Sci 1982;65:2063-69.

37. Sanders ME, Walker DC, Walker KM, et al. Performance of commercial cultures in fluid milk applications. J Dairy Sci 1996;79:943-55.

38. Klaenhammer TR. Microbiological considerations in selection and preparation of Lactobacillus strains for use as dietary adjuncts. J Dairy Sci 1982;65:1339-49.

39. Goldin B, Gorbach SL. Alterations in fecal microflora enzymes related to diet, age, Lactobacillus supplements, and dimethylhydrazine. Cancer 1977;40:2421-26.

40. Isolauri E, Sütas Y, Kankaanpää P, et al. Probiotics: effects on immunity. Am J Clin Nutr 2001;73(suppl):444S-50S.

41. Rasic JLj, Kurmann JA. Bifidobacteria and Their Role. Boston: Kirkhauser Verlag; 1983.

42. Gibson GR, Roberfroid MB. Dietary modulation of the human colonic microbiota: introducing the concept of prebiotics. J Nutr 1995;125:1401-12. 